\title{
Shedding Light on the Molecular Pathology of Amyloid Plaques in Transgenic Alzheimer's Disease Mice using Multimodal MALDI Imaging Mass Spectrometry
}

Kaya I, Zetterberg H, Blennow K and Hanrieder J

Department of Psychiatry and Neurochemistry, Sahlgrenska Academy at the University of Gothenburg, Mölndal, Sweden

Department of Chemistry and Molecular Biology, University of Gothenburg, Sweden

Clinical Neurochemistry Laboratory, Sahlgrenska University Hospital, MöIndal, Sweden

Department of Molecular Neuroscience, Institute of Neurology, University College London, UK UK Dementia Research Institute at UCL, London, UK

Department of Chemistry and Chemical Engineering, Chalmers University of Technology, 412 96 Gothenburg, Sweden

\section{Corresponding Author}

Assoc. Prof. Jörg Hanrieder, PhD

Department of Psychiatry and Neurochemistry, Sahlgrenska Academy at the University of Gothenburg, Mölndal Hospital, House V, Biskopsbogatan 27, SE-43180 Mölndal, Sweden ih@gu.se;+46-31-3432377

\section{ABSTRACT}




\section{INTRODUCTION}

Alzheimer's disease is a fatal neurodegenerative disorder originally characterized by three neuropathological features including intracellular neurofibrillary tangles (NFTs) consisting of hyperphosphorylated tau protein ${ }^{1}$, senile plaques formed by aggregated amyloid $\beta$ peptides 
$(A \beta)^{2}$ and lipid granule accumulations (lipoid granules) in neuroglia. ${ }^{3,4}$ According to the amyloid hypothesis ${ }^{2}$, accumulation of $A B$ is the primary influence triggering $A D$ pathogenesis and the rest of the disease process. ${ }^{5} \mathrm{~A} \beta$ aggregates into oligomers, which can be of various sizes, and forms diffuse and neuritic plaques in the brain parenchyma, leading to loss of synapses and neurons. ${ }^{6,7}$ However, molecular mechanisms of how monomeric $A \beta$ peptides are converted to neurotoxic oligomers and further to fibrils rich in cross $\beta$-sheet motifs are indeterminate.

Alois Alzheimer's originally reported pathologic hallmark of AD; lipoid granules in neuroglia ${ }^{4}$ and strong clinical evidence demonstrated in the ensuing years suggest an aberrant lipid homeostasis associated with $A D$ pathology along with peptide and protein centric mechanisms, in part for the following reasons.$^{3,8}$ All APP-cleaving secretases as well as APP are transmembrane proteins, suggesting a direct relationship of amyloid pathology with neuronal membrane lipids. Importantly, growing evidence indicates that amyloidogenic processing of APP occurs in lipid rafts, largely because functionally active pools of BACE1 and $\mathrm{Y}$-secretase are present in these cholesterol- and sphingolipid rich membrane micro domains. ${ }^{9}$ It is also known that $A \beta$ exerts its cytotoxic effects, at least partly, by perturbating cellular membranes, potentially through the modulation of the activity of phospholipases, such as $\mathrm{PLA}_{2}{ }^{10}$ and PLC. ${ }^{11}$ On the other hand, lipid species, such as ganglioside GM1 was found to trigger $A \beta$ aggregation. ${ }^{12}$ Indeed, a major risk factor for sporadic $A D(S A D)$ is the $\varepsilon 4$ allelic variant of the apolipoprotein $\mathrm{E}(A P O E)$ gene, which encodes a protein in cholesterol metabolism and lipid transport further linking AD pathology to lipids ${ }^{13}$. It is thus clear that there is an interrelating molecular pathology of neuronal lipids, peptides and proteins associated with AD pathology. ${ }^{8,14,15}$ Therefore, a comprehensive lipidome and peptidome/proteome analysis would provide a basis for further understanding of associated molecular changes in $A D$ pathogenesis.

Advances in lipidomics and peptidomics/proteomics, particularly through the use of electrospray ionization mass spectrometry (ESI-MS), has facilitated an accurate profiling of AD-associated changes in lipids such as cholesterol ${ }^{16}$, plasmalogens ${ }^{17}$, sphingolipids ${ }^{18}$, 
amyloid peptides ${ }^{19}$ and protein species such as apoE4 protein ${ }^{20}$ in brain tissue. However, these studies were performed using brain tissue extracts where spatially confined changes are convoluted, restricting the direct correlation of molecular information with amyloid plaques which are scenes of $A D$ pathogenesis. Therefore, advanced chemical imaging techniques are required to characterize disease pathology in situ, such as imaging mass spectrometry (IMS). ${ }^{21}$ This technique is suited to probe amyloid plaque-associated molecular pathology in specific brain regions. ${ }^{22-31}$ In particular, MALDI-IMS was shown to be a powerful technique to probe amyloid plaque-associated $A \beta$ isoforms and neuronal lipids in transgenic mice and postmortem human AD brains. ${ }^{23,27-31}$

Although the vast majority of $A D$ cases are classified as late-onset $S A D$, rare but highly penetrant autosomal mutations in genes encoding APP and presenilin 1 and presenilin 2, the catalytic components of the $y$-secretase, result in early-onset familial $A D(F A D)^{32}$, sharing certain pathological characteristics with SAD. Therefore, transgenic animal models of AD can serve as model systems for more thorough investigations of the aggregation mechanisms, cell signaling and metabolic pathways in vivo. For example, the mouse model (tgArcSwe), carrying both the Arctic (E693G) and the Swedish (K670M/N671L) mutation, displays extensive A $\beta$ deposition with an early onset age of $5-6$ months $^{33}$, and is therefore a well-suited model system to study $A \beta$ plaque pathology.

In this communication, we attempted to shed a light on the molecular pathology of individual hippocampal $A \beta$ plaques in 18 month-old tgArcSwe mice, which can give insight into the pathophysiological processes leading to disease states in AD. We utilized multimodal MALDIIMS analysis on the same tissue sections to sketch a high-throughput molecular architecture of individual amyloid plaques. Subsequent high-spatial resolution $(10 \mu \mathrm{m})$ dual polarity lipid and peptide/protein MALDI-IMS analysis on the same regions of single tissue sections allowed correlation of the ion images of lipids including sphingolipids, phospholipids, lysophospholipids with multiple amyloid $\beta$ isoforms at cellular levels in specific brain regions such as hippocampus of $\operatorname{tg} A r c S w e$, where extensive amyloid plaque accumulations were observed. 


\section{EXPERIMENTAL SECTION}

\section{METHODS}

Chemicals and Reagents. All chemicals for matrix and solvent preparation were pro-analysis grade and obtained from Sigma-Aldrich (St. Louis, MO), unless otherwise specified. TissueTek optimal cutting temperature (OCT) compound was purchased from Sakura Finetek (AJ Alphen aan den Rijn, the Netherlands). The $d d \mathrm{H} 2 \mathrm{O}$ was obtained from a MilliQ purification system (Merck Millipore, Darmstadt, Germany).

Animals, Tissue Sampling and Sectioning. Transgenic mice $(n=3), 18$ months of age, with the Arctic (E693G) and Swedish (K670N, M671L) mutations (tgArcSwe) of human APP were reared ad libitum at the animal facility at Uppsala University under a 12/12-hlight/dark cycle. The animals were anesthetized with isoflurane and killed by decapitation. The brains were dissected quickly with 3 min postmortem delay and frozen on dry ice. All animal procedures were approved by an ethical committee and performed in compliance with national and local animal care and use guidelines (DNr \#C17/14 at Uppsala University). Frozen tissue sections (12 $\mu \mathrm{m}$ thick) were cut in a cryostat microtome (Leica CM 1520, Leica Biosystems, Nussloch, Germany) at $-18^{\circ} \mathrm{C}$, and collected on special-coated, conducting glass slides (indium tin oxide, Bruker Daltonics, Bremen, Germany) and stored at $-80^{\circ} \mathrm{C}$.

Sample Preparation and Matrix Application Strategies. Prior to matrix deposition by sublimation, tissue sections were thawed in a desiccator for $\sim 30$ minutes under reduced pressure (SpeedVac, Eppendorf, Hamburg, Germany). For teaching of the navigation points in Flex Imaging v3.0 software, we used a glass cutter pen (Starwalker, Montblanc, Germany) to leave sharp-edged cross signs on tissue-mounted indium tin oxide (ITO) glass. The sections were scanned in a slide scanner (PathScan Enabler IV, Electron Microscopy Sciences, Hatfield, PA, USA) to visualize the specific brain regions. Matrix deposition for lipid analysis was carried out using a vacuum sublimation apparatus (Sigma-Aldrich) comprising an inner 
flat top and an outer bottom attached to each other by an O-ring-sealed flange. The chamber was connected to a rough pump attached to a digital vacuum gauge controller and placed in a heated sand bath $\left(\mathrm{SiO}_{2}, 50-70\right.$ mesh particle size, Sigma-Aldrich) on a hot plate (C-MAG HP 4, IKA Werke GmbH \& Co. KG, Staufen, Germany) connected to an electronic contact thermometer (ETS-D5, IKA Werke GmbH \& Co. KG, Staufen, Germany). Sublimation was performed by the following steps. First, vacuum-dried ( 20 minutes) ITO-glass slides with the thaw-mounted mouse brain tissues were attached to the flat top of the chamber using doublesided heat conductive copper tape (12mmx16.5m copper, 3M, United States). Then, 300 mg of 1,5-diamnionapthalene (1,5-DAN) matrix powder was spread evenly on the outer bottom of the sublimation chamber, which was then attached to the top using the O-ring seal. A vacuum of 0.8 mbar was provided by a membrane pump and the system was allowed to equilibrate under vacuum for a period of $\sim 20$ minutes. The cooler was filled with ice slush $\left(\geq 0{ }^{\circ} \mathrm{C}\right)$ for condensation of the matrix on the sample slides. The process was halted by removing the sublimation apparatus from the sand bath and closing the vacuum valve. The sublimation apparatus was allowed to slowly re-equilibrate to atmospheric pressure before removal of the sample plate. The amount of deposited matrix on ITO glass was determined using a highprecision scale (AX224, Sartorius, Göttingen, Germany) weighing the amount before and after the sublimation experiments. The sublimation protocol was optimized with respect to temperature, deposition time and total amount of deposited matrix to obtain the best detection efficiency for lipids on mouse brain tissue. With this setup, the optimum matrix layer was found to be $120 \mu \mathrm{g} / \mathrm{cm}^{2}$ to give the best lipid signals. We used optimized sublimation conditions: 20 minutes at a temperature of $130^{\circ} \mathrm{C}$ under a stable vacuum of $0.8 \mathrm{mbar}$. Prior to protein analysis, tissue sections were washed $2 \times 100 \%$ ethanol $(\mathrm{EtOH})(30$ s) to get rid of the remaining $1,5-$ DAN matrix molecules on the tissue surface. Lipids and salts on the tissue surface were washed away in sequential washes of $70 \% \mathrm{EtOH}$ (30s), $100 \% \mathrm{EtOH}$ (30s), Carnoy's fluid (6:3:1 EtOH:chloroform:acetic acid) (2min), $100 \% \mathrm{EtOH}(30 \mathrm{~s}), \mathrm{H}_{2} \mathrm{O}$ with $0.2 \%$ TFA (30s), and 100\% $\mathrm{EtOH}(30 \mathrm{~s}) .{ }^{34}$ For teaching of the navigation points we used the same sharped-edged cross points created with the glass cutter pen prior to lipid analysis to minimize the repositioning error 
between lipid and protein imaging analysis. For protein MALDI-IMS, 2,5dihydroxyacetophenone (2,5-DHA) matrix compound was applied using a TM Sprayer (HTX Technologies, Carrboro, NC, USA) combined with a HPLC pump (Dionex P-580, Sunnyvale, CA, USA). Before every spraying experiment, the corresponding pump valve was cleaned from big bubbles using a syringe and $\sim 10$ minutes purge was applied to get rid of the little bubbles. Then the pump was kept running at $100 \mu \mathrm{l} / \mathrm{min}$ using a $70 \%$ ACN pushing solvent with isocratic pressure for 3 hours. $15 \mathrm{mg} / \mathrm{ml}$ 2,5-DHA in $60 \% \mathrm{ACN} / 1 \% \mathrm{CH}_{3} \mathrm{COOH} / 1 \%$ TFA solution was sprayed over the tissue sections using instrumental parameters including nitrogen flow of 10 psi, spray temperature of $75^{\circ} \mathrm{C}$, nozzle height of $40 \mathrm{~mm}, 8$ passes with offsets and rotations, a spray velocity of $1300 \mathrm{~mm} / \mathrm{min}$. These parameters were optimized to avoid analyte delocalization on the tissue surface, while keeping the optimum incorporation of matrix and analyte molecules for an efficient desorption/ionization.

MALDI-IMS Analysis. Imaging MS analysis of tissue sections was performed on a MALDITOF/TOF UltrafleXtreme mass spectrometer equipped with SmartBeam II Nd:YAG/355 nm laser operating at $1 \mathrm{kHz}$ providing a laser spot diameter down to $\sim 10 \mu \mathrm{m}$ for the 'minimum' focus setting (Bruker Daltonics). Measuring the absolute laser pulse energy of a smartbeam II laser is challenging in UltrafleXtreme systems, as such a measurement would require a reference measurement inside the instrument, which would require modification of the instrument hardware. Moreover, SmartBeam lasers do not have a flat-top shaped beam energy profile, but a rather highly structured beam energy profile, which makes it a hard task to measure the exact value of laser fluence at a flat target surface. ${ }^{35}$ Therefore, rather than measuring the laser pulse energy, we provide detailed information about the laser pulse energy settings as previously indicated for lipid analysis in our previous communication; ${ }^{30}$ global laser attenuator setting was kept stable at $10 \%$ throughout all the experiments and the laser focus set to minimum for $10 \mu \mathrm{m}$ and small for $30 \mu \mathrm{m}$ spatial resolution analysis. Attenuator offset and attenuator range settings were $40 \%$ and $10 \%$ for all the analysis. As SmartBeam II lasers lose 
some energy output over lifetime, it is necessary to specify the laser shot count of the instrument unit used for this experimentation, which was about 225696k (in 12 months age). For dual polarity lipid analysis, profiling and imaging data acquisitions were performed in reflective ion mode under optimized delayed extraction conditions in a mass range of 300-3000 Da. First, IMS data acquired with 5 laser shots/per pixel point with a source accelerating voltage of $20 \mathrm{kV}$ in negative polarity which was followed by 50 laser shots/pixel $\mathrm{p}$ oint in positive polarity with a source accelerating voltage of $25 \mathrm{kV}$. The detector gain value was kept stable at $2626 \mathrm{~V}$ for both ionization modes. A mass resolution of $\mathrm{M} / \Delta \mathrm{M} 20000$ was achieved in the mass window of lipids (i.e., 650-1000 Da). External calibration was carried out using peptide calibration standard I (Bruker Daltonics). Image data were reconstructed and visualized using Flex Imaging v3.0 (Bruker Daltonics).

For peptide/protein analysis on the same imaging region of the same tissue section, global laser attenuator was increased to $35 \%$ while keeping attenuator offset and range settings the same. Profiling and imaging data acquisitions were performed in linear positive ion mode in a mass range of 2000-20000 Da. Approximately 50 laser shots/raster spot were acquired with 1 $\mathrm{kHz}$ repetition rate.

Identification of amyloid $\beta$ plaque-associated lipids and $A \beta 1-40 \mathrm{~m} / \mathrm{z} 4257.6$ was achieved by examining MS/MS spectra obtained in LIFT-TOF/TOF mode as previously described. ${ }^{29,31}$ MALDI-LIFT (MS/MS) spectra were collected directly from plaque deposits in LIFT mode until a satisfactory convolution of fragment spectra was achieved. Lipid classifications were determined by comparing mass accuracy data and fragment spectra with the LIPID MAPS database (Nature Lipidomics Gateway, www.lipidmaps.org) and previous results obtained on $\operatorname{tg} A r c S w e$ brain tissue sections for the lipid species have already been reported. ${ }^{29,31}$

\section{RESULTS AND DISCUSSION}

High-Resolution, Dual Polarity MALDI-IMS: Enhanced Hippocampal Lipid Spectral Quality and Spatial Molecular Information 
High resolution MALDI-IMS is a strong approach to interrogate the spatial distribution profiles of biological molecules associated with spatially-confined histopathological features in neuroscience. ${ }^{30,34,36} 1,5$-DAN was previously demonstrated to be a highly efficient MALDI matrix via sublimation matrix coating for brain lipids in both negative and positive polarities compared to several commonly used matrix compounds. ${ }^{36}$ It is also reported that 1,5 -DAN gives enhanced spectral quality of brain lipid species in a wide spectral range with few number of low-energy laser pulses at high spatial resolutions. ${ }^{30}$ Furthermore, ion signals are distinctly diverse and intense in negative polarity compared to positive polarity ${ }^{30,36}$ which can be attributed to distinct reductive properties and radical ion transfer abilities of $1,5-\mathrm{DAN}^{37}$ With respect to enhanced correlated lipidome analysis using 1,5-DAN sublimation MALDI-IMS approach, it was also demonstrated that dual polarity lipid analysis can be performed on the same pixel points at high spatial resolutions. ${ }^{31}$ Here, this approach was utilized to reveal a comprehensive lipidome of hippocampus region of $\operatorname{tg}$ ArcSwe mice brain (Figure 1). Due to the limitations of the speed of data acquisition in UltrafleXtreme MALDI-TOF/TOF instruments at high spatial resolutions which is restricted by the vacuum stability of 1,5 -DAN matrix molecule ${ }^{36}$, experiments were performed on the hippocampal areas of tgArcSwe coronal brain tissue sections where extensive amyloid plaque aggregates were revealed by previous amyloid specific IHC staining and/or MALDI-IMS studies. ${ }^{29,33,38}$ Enhanced ionization at highspatial resolutions using low-energy few number of laser shots along with the minimized matrixderived signals revealed a high throughput hippocampal lipid spectral quality in transgenic AD mice (tgArcSwe) brain tissue in both polarities and on the same pixel points (Figure1).

In negative ionization mode, matrix related signals were minimized down to $320 \mathrm{Da}$. This yielded an enhanced low-molecular weight lipid signals (320-620 Da) otherwise would be suppressed and/or interfered by matrix-derived $\operatorname{signals}^{30}$, along with the lipids in phospholipid range (620-1000 Da) and relatively high-molecular weight species, gangliosides (1000-2000 Da) (Figure 1la). Therefore, this yielded spatially well-resolved, lipid signals in a wide spectral range as indicated as ion images of various lipid species such as lysophosphatidic acids (LPA, 
m/z 409.3), ceramides (Cer 18:0 m/z 564.6), ceramide phosphates (Cer $P, \mathrm{~m} / \mathrm{z}$ 644.6), phosphatidylethanolamines (PE 40:6, m/z 790.6), phosphatidylinositols (PI 38:4, 885.6), sulfatides (ST 24:0, 888.6) and gangliosides (GM3 18:0, m/z 1179.7, GM1 18:0, m/z 1544.8) (Figure $1 \mathrm{lb}$ ). In positive ion mode, matrix related signals were minimized down to $420 \mathrm{Da}$. This allowed us to observe low-molecular weight lysophosphatidylcholines, otherwise would be suppressed and/or interfered by matrix-derived signals ${ }^{30}$ (Figure 1lla). Moreover, intense phospholipid signals, mainly phosphatidylcholines, were observed in the mass range of 650$900 \mathrm{Da}$ along with the dimer peaks of phospholipids (1400-1700 Da) as previously observed in MALDI-TOF-MS ${ }^{39}$ (Figure 1lla). As a result, spatially well-resolved ion images of Iysophophatidycholines (LPC 16:0, m/z 496.3, LPC 18:1, m/z 522.3, LPC 18:0, m/z 524.3, LPC 20:4, m/z 544.3) and phosphatidycholines (PC 32:0, m/z 734.6, PC 34:1, m/z 760.6, PC 36:4, $\mathrm{m} / \mathrm{z}$ 782.6, PC 38:6, m/z 806.6) were observed (Figure 1llb), which can be simultaneously correlated with ion images obtained in negative polarity on the same pixel points (Figure $1 \mathrm{lb}$ ). 


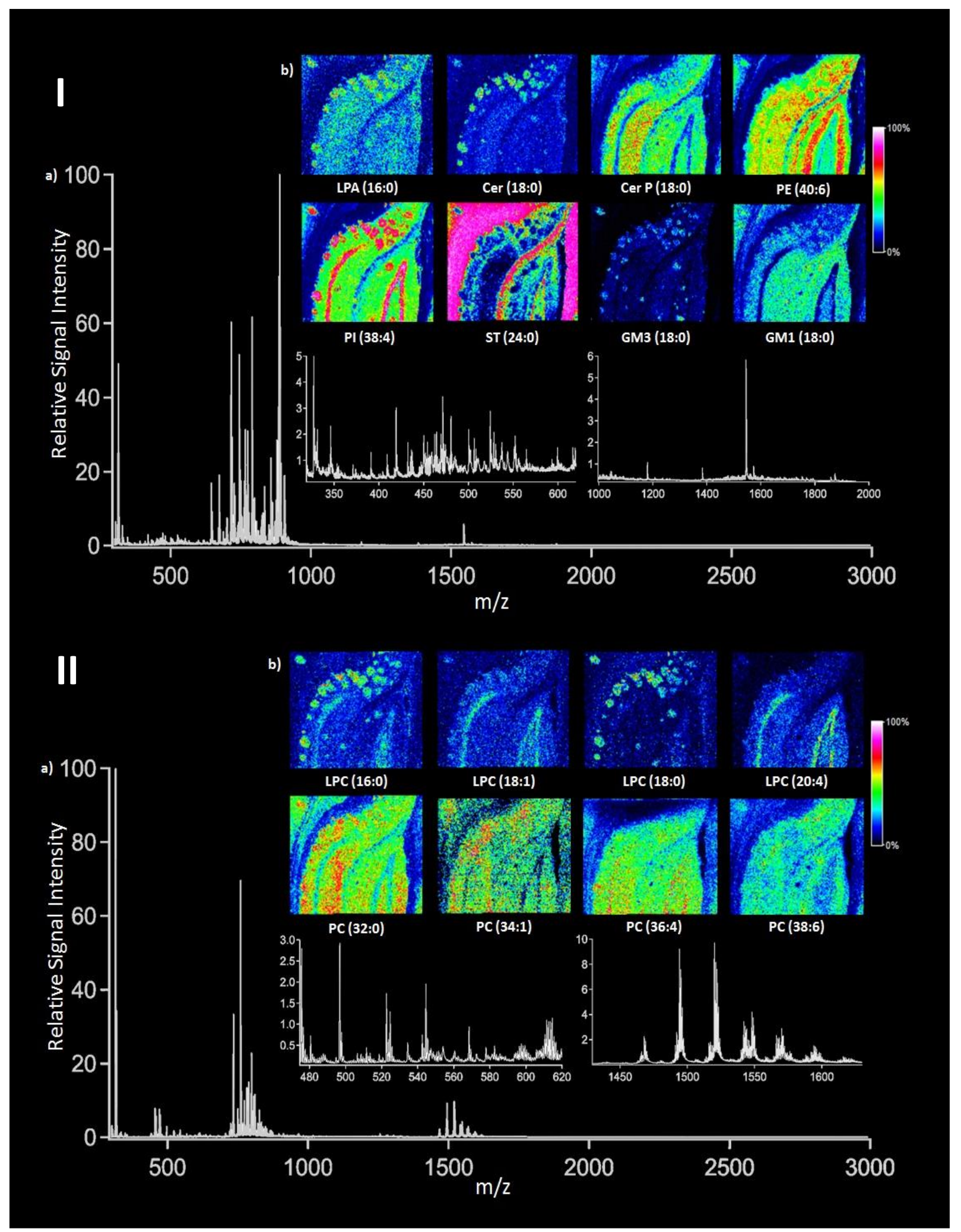

Figure 1. MALDI-MS spectra acquired subsequently in I a) negative and II a) positive ion mode on the same pixel points from hippocampal region of a coronal tissue section of tgArcSwe mouse brain. I a) Inset spectra in negative ion mode from left to right indicates low molecular weight (320-620 Da) lipid 
species and relatively high-molecular weight (1000-2000) lipid species, respectively. I b) lon images of lipid species such as lysophosphatidic acids (LPA, m/z 409.3), ceramides (Cer 18:0 m/z 564.6, Cer P, m/z 644.6), phosphatidylethanolamines (PE 40:6, m/z 790.6), phosphatidylinositols (PI 38:4, 885.6), sulfatides (ST 24:0, 888.6) and gangliosides (GM3 18:0, m/z 1179.7, GM1 18:0, m/z 1544.8). II a) Inset spectra in positive ion mode from left to right indicates low molecular weight (420-620 Da) lipid species and relatively high-molecular weight (1400-1700) phospholipid dimers, respectively. II b) Ion images of lipid species such as lysophosphatidylcholines (LPC 16:0, m/z 496.3, LPC 18:1, m/z 522.3, LPC 18:0, $\mathrm{m} / \mathrm{z}$ 524.3, LPC 20:4, m/z 544.3) and phosphatidycholines (PC 32:0, m/z 734.6, PC 34:1, m/z 760.6, PC 36:4, m/z 782.6, PC 38:6, m/z 806.6).

On the other hand, enhanced ionization of lipid species at high-spatial resolutions allowed us to observe pathological lipid changes not only brain region-specific but also directly correlated to the plaque aggregates (Figure 2), whose amyloid $\beta$ identities were demonstrated by subsequent peptide imaging on the same imaging sequence in hippocampus of tgArcSwe (Figure 3). To further demonstrate the relevance of high-resolution to probe plaque-associated lipid changes, we compared ion images obtained at $30 \mu \mathrm{m}$ and $10 \mu \mathrm{m}$ spatial resolutions in equivalent hippocampal regions of subsequent tissue sections (Figure 2). For example, ion images of ceramide phosphates (CerP 18:0, 644.6), phosphatidylethanolamines (PE 40:6, m/z 790.6), and phosphatidylinositols (PI 38:4, m/z 885.6) display distinct amyloid-plaque associated localizations which can be observed explicitly at a spatial resolution of $10 \mu \mathrm{m}$

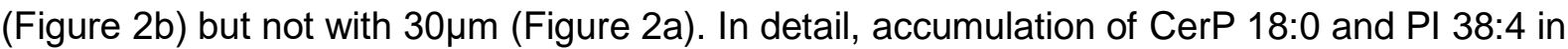
the cores and periphery of certain amyloid plaques, respectively, along with the mild reduction of PE 40:6 in the certain amyloid plaques in spatially well-resolved hippocampal regions can be luculently observed at $10 \mu \mathrm{m}$ spatial resolutions (Figure $2 \mathrm{~b}$ ). 


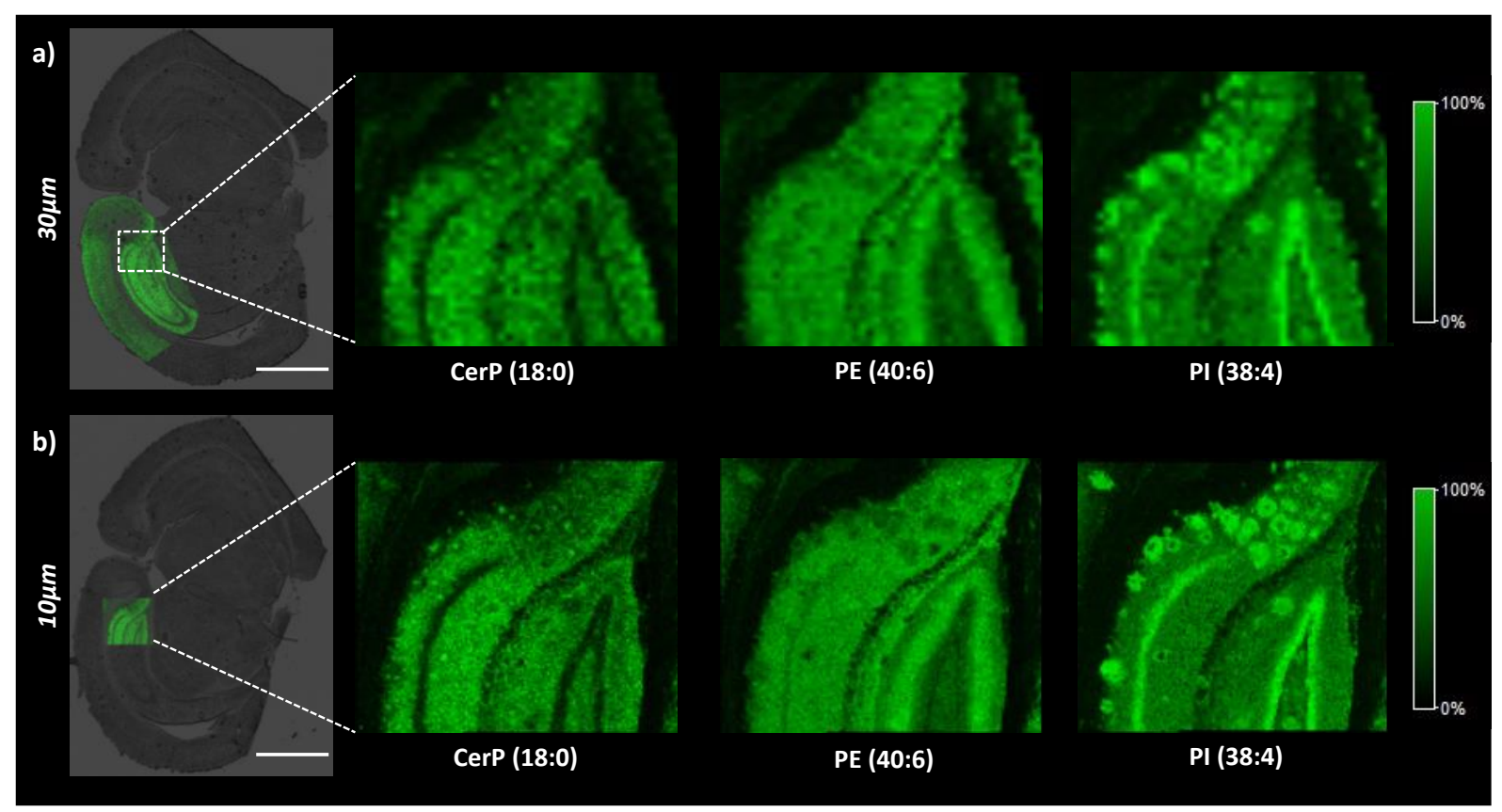

Figure 2. MALDI-IMS analysis of tgArcSwe mice hippocampal lipids of coronal mouse brain tissue section. Ion images of Ceramide phosphates (CerP 18:0, m/z 644.6), phosphatidylethanolamines (PE 40:6, $\mathrm{m} / \mathrm{z} 790.6$ ), phosphatidylinositols (PI 38:4, 885.6) were obtained in negative ion mode at spatial resolutions of a) $30 \mu \mathrm{m}$ b) $10 \mu \mathrm{m}$. Scale bars: $2 \mathrm{~mm}$.

\section{Amyloid Plaque Identification by Subsequent High-resolution Peptide MALDI-IMS on the}

\section{Same Tissue Section}

1,5-DAN via sublimation allows enhanced ionization of lipid species without the need of high laser pulse energies. ${ }^{30}$ This "gentle" MALDI-IMS methodology preserves the tissue morphology by minimizing the thermal and mechanical nanosecond laser ablation effects during the laser desorption/ionization process ${ }^{30}$ and therefore allows successful fluorescent immunohistochemistry ${ }^{30}$ or peptide/protein MALDI-IMS ${ }^{31}$ on the same tissue section. In order to demonstrate the amyloid identity of lipid aggregates in the same hippocampal regions, subsequent MALDI-IMS was applied for peptide/protein analysis on the same tissue section. This approach was demonstrated to be an alternative and superior strategy to previously applied amyloid specific immunohistochemistry and fluorescent staining experiments as it allows correlation of multiple $A \beta$ isoform ion images with lipid ion images in dual polarity. ${ }^{31}$ Therefore, we employed tri-modal MALDI-IMS paradigm for negative- and positive ion mode 
lipid analysis and subsequent peptide/protein ion imaging on the same tissue section at $30 \mu \mathrm{m}$ and $10 \mu \mathrm{m}$ spatial resolutions (Figure 3$)$. High spatial resolution $(10 \mu \mathrm{m})$ tri-modal MALDI-IMS revealed an accurate correlation of dual polarity lipid ion images such as phosphatidylinositols (PI 38:4, 885.6) in negative ion mode and lysophosphatidylcholines (LPC 16:0, m/z 496.3) in positive ion mode with the ion image of amyloid peptide $A \beta$ 1-40 (Figure $4 \mathrm{~b}$ ) to individual plaques, whereas the correlation was not conclusive at $30 \mu \mathrm{m}$ spatial resolution (Figure 3a). These results are potentially important as this high spatial resolution method allows correlation of tri-modal ion images to not only brain regions but also to individual plaque features which are spatially well-resolved (Figure 3b).

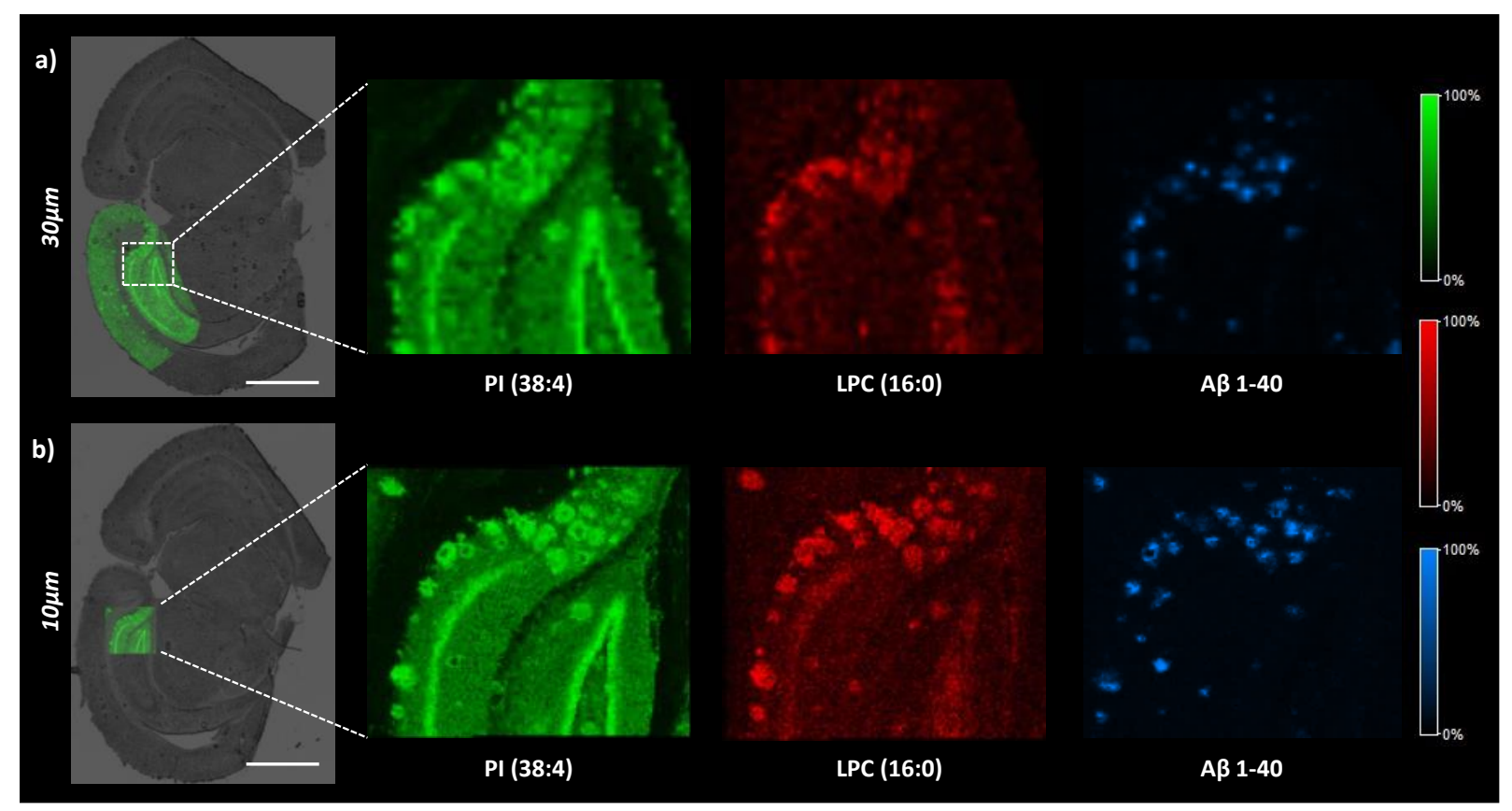

Figure 3. Trimodal MALDI-IMS analysis of tgArcSwe mice hippocampal lipids and peptides on the same tissue section. Ion images of phosphatidylinositols (PI 38:4, $\mathrm{m} / \mathrm{z} 885.6$ ) in reflective negative ion mode, lysophosphatidylcholines (LPC 16:0, $\mathrm{m} / \mathrm{z}$ 496.3) in reflective positive ion mode and amyloid peptide $(A \beta 1-40, \mathrm{~m} / \mathrm{z} 4257.6)$ in linear positive ion mode were obtained at a) $30 \mu \mathrm{m}$ and b) $10 \mu \mathrm{m}$ spatial resolutions. Scale bars: $2 \mathrm{~mm}$.

All in all, these methodologies suggest a high throughput investigation of lipid microenvironment of individual $A \beta$ plaques and elucidate their plaque-associated alterations. 


\section{Amyloid Plaque-Associated Alterations of Lipids in tgArcSwe Mice Brain Revealed by Multimodal MALDI-IMS: Insights into the Molecular Pathology}

Amyloid plaque formation is a dynamic process which was regulated by several enigmatic biochemical and biophysical processes. Most of the lipid classes were shown to be crucial players in $A D$ pathogenesis including amyloid plaque pathology. ${ }^{8,15}$ Based on the published studies, one could have in view following main mechanisms for the action of lipids in amyloid pathology and senile plaque formation: Firstly, the interaction of amyloid peptides and/or amyloid oligomers with the cellular membranes effecting the membrane lipid homeostasis and/or effecting the organization and physicochemical properties of the membrane bilayer which alters APP processing. ${ }^{11,40}$ Secondly, the functional roles of peptides/proteins such as $A \beta$ and APP Intracellular domain (AICD) in the regulation of lipid metabolism pathways either directly or by effecting the gene expressions encoding for APP, BACE1, the A $\beta$-degrading protease neriplysin (NEP) as well as several enzymes involved in lipid metabolism. ${ }^{15,41-45}$ Bidirectionally, lipid-peptide/protein interactions, whereby changes in lipids modulate the function of target proteins such as APP and APP-cleaving secretases. ${ }^{46,47}$ Finally, catalyzer

and/or interfacial role of lipids in amyloid oligomerization and fibrillization. ${ }^{12,48,49}$ Considering the heterogeneity of lipid molecules and complex bidirectional link between lipids and amyloid pathology, it is expectative to observe amyloid plaque-associated alterations of several lipid classes in tgArcSwe mice brain tissue. Here, high-resolution, highthroughput MALDI-IMS reveals a number of amyloid plaque-associated alterations of lipids including sphingolipids, phospholipids and lysophospholipids, which can give insights in to the molecular pathology of $A D$ pathogenesis.

Sphingolipids including ceramides, sphingomyelins, and glycosphingolipids (GSLs) are major components of lipid rafts, playing a number of crucial parts in cell functions. ${ }^{50}$ Growing evidence indicating that the amyloidogenic processing of APP occurs in lipid rafts ${ }^{9}$ closely links sphingolipids to amyloid pathology. Moreover, the APP-processing product AICD downregulates the gene expression encoding for serine palmitoyl-CoA (SPT) enzyme which 
initiates the sphingolipid biosynthesis. ${ }^{42}$ In addition, a number of studies have been reported on the alterations of sphingolipids in post-mortem human $A D$ and transgenic $A D$ mice brain tissue and their potential to modulate APP processing and $A \beta$ aggregation. ${ }^{51,52}$

High-spatial resolution MALDI-IMS was performed on a hippocampal and adjacent cortical regions of a coronal tgArcSwe mice brain tissue section (Figure 4). Inspection of ion images in reflective negative ion mode revealed plaque-associated alterations of sphingolipids such as the accumulation of ceramides (Cer 14:0 m/z 508.3, Cer 18:0 m/z 564.6), and gangliosides (GM3 18:0, m/z 1179.8, GM3 20:0, m/z 1207.8, GM2 18:0, m/z 1382.8 ) along with the depletion of sulfatides (ST 18:0, m/z 806.8, ST 22:0, m/z 862.8, ST 22:0(OH), m/z 878.8, ST 24:0, m/z 890.8, ST 24:0 (OH), 906.8 m/z). Further, subsequent MALDI-IMS for peptides was performed on the same region of the tissue section to identify the amyloid deposits. An intense accumulation of $A \beta 1-40, \mathrm{~m} / \mathrm{z} 4257.6$ (Figure $4 \mathrm{~m}$ ) along with the other amyloid isoforms (AB 137, $\mathrm{m} / \mathrm{z} 4002.7, A \beta$ 1-38, $\mathrm{m} / \mathrm{z} 4060.3$ and $A \beta$ 1-42 m/z 4441.2) (Figure 4p) all colocalized with spatial lipid alterations.

Amyloid plaque-associated alterations of certain sphingolipids were previously described for the same transgenic mice (tgArcSwe) using MALDI-IMS and subsequent IHC and fluorescent staining on the same tissue section. ${ }^{29}$ Here, high spatial-resolution multimodal MALDI-IMS reveals amyloid-plaque associaited alterations of several sphingolipid species all correlated to multiple $A \beta$ isoforms at $10 \mu \mathrm{m}$ spatial resolution (Figure 4). Previously, shotgun lipidomics consistently revealed substantial decrease of sufatides and large content increase of ceramides in post-mortem human AD brain extracts relative to cognitively normal age-matched controls. ${ }^{18}$ Our data indicates both hydroxylated and non-hydroxylated sulfatides, irrespective of their fatty acid (FA) moeity, depletes on the amyloid plaques (Figure 4e-i). Interestingly, ST $(18: 0)$ species shows lesser depletion relative to the longer chain sulfatides in the center of the plaques and a clear elevation on the vicinity (Figure 4e). 


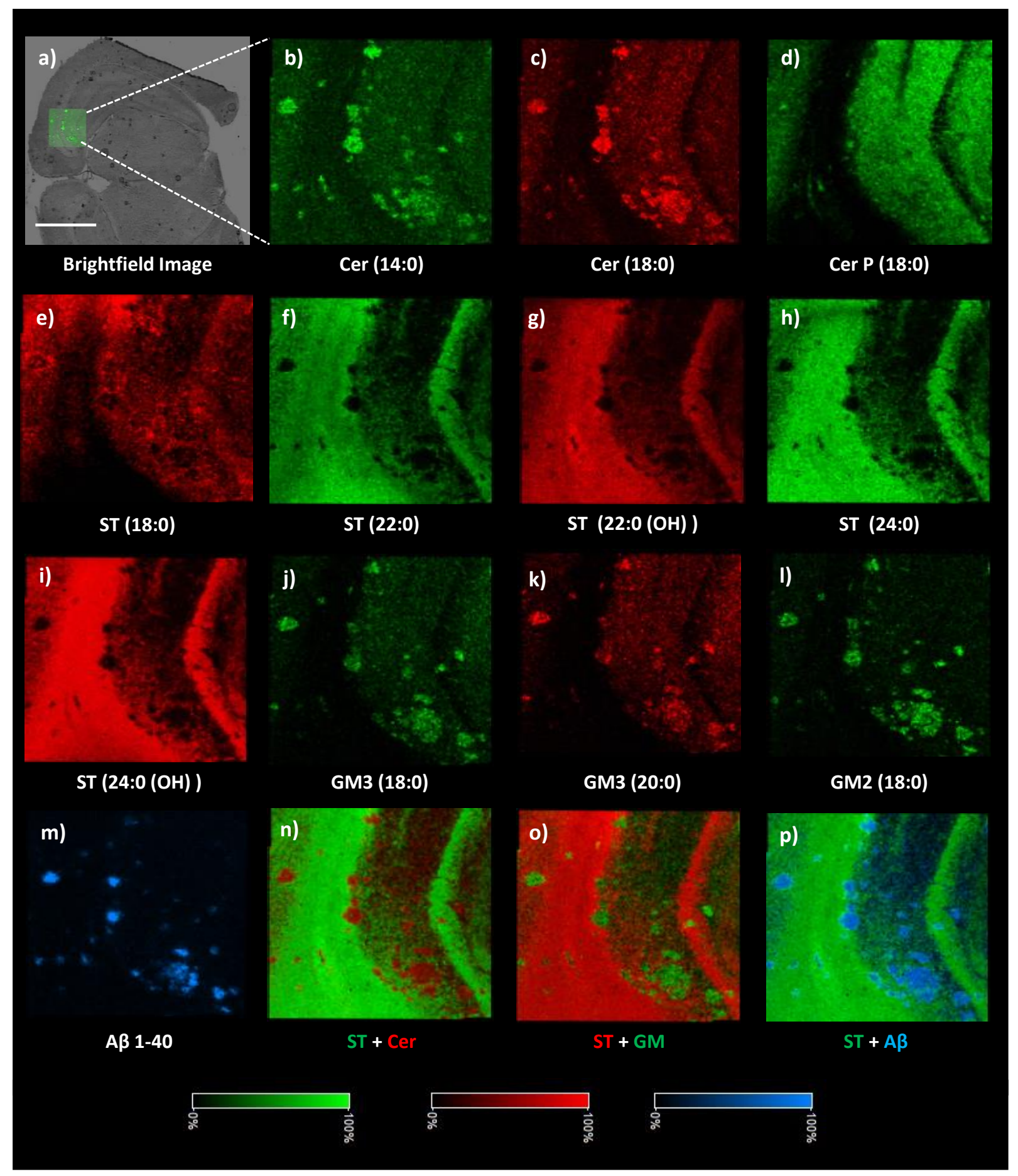

Figure 4. Amyloid plaque-associated alterations of sphingolipids correlated with amyloid peptide isoforms revealed by Multimodal MALDI-IMS on the same tissue section of tgArcSwe mouse brain.a) brightfield image of coronal brain tissue section of tgArcSwe b) Cer 14:0, m/z 508.3, c) Cer 18:0, m/z 564.6, d) Cer P 18:0, m/z 644.6, e) ST18:0, m/z 806.8, f) ST 22:0, m/z 862.8, g) ST:22:0 (OH), m/z 878.8, h) ST 24:0, m/z 890.8, i) ST 24:0 (OH), m/z 906.8, j) GM3 18:0, m/z 1179.8, k) GM3 20:0, m/z 1207.8, I) GM2 18:0, m/z 1382.8 m) A 1-40, m/z 4257.6, n) merged ion images of sulfatides (green) and ceramides (red), o) merged ion images of sulfatides(red) and gangliosides (green), p) merged ion 
images of sulfatide(green) and amyloid peptides isoforms (blue) (A $\beta$ 1-37, $\mathrm{m} / \mathrm{z} 4002.7, A \beta$ 1-38, $\mathrm{m} / \mathrm{z}$ 4060.3 and $A \beta$ 1-42, $\mathrm{m} / \mathrm{z}$ 4441.2). Scale bar: $2 \mathrm{~mm}$.

Further, elevation of ceramides (Cer 14:0, Cer 18:0) were correlated with the depletion of all the sulfatides (Figure $4 n$ ) and all together correlated to amyloid isoforms (Figure $4 m, 4 p)$. On the other hand, ceramide phosphates (CerP 18:0, m/z 644.6) showed an inconsistent plaqueassociated distribution. They were elevated in the cores and depleted on the edges of certain plaques (Figure $2 b$, Figure $4 d$ ), which might be attributed to the structural heterogeneity and therefore the distinct molecular composition of amyloid plaques.

Sulfatides are one of the main consitutents of the myelin sheath surrounding axons in the CNS and mainly synthesized by oligodendrocytes playing key roles in the regulation of oligodendrocyte maturation and myelin formation. ${ }^{53,54}$ Ceramides are the central components in sphingolipid metabolism and serve as the backbones to generate sphingomyelin (SM) and GSLs including sulfatides and gangliosides. Early reports indicated the role of apoE in both CNS and peripheral nervous system which is the regulation of sulfatide level. ${ }^{55}$ Further, it has been hypothesized that the alterations of $A P O E \varepsilon 4$ allele-associated sphingolipid homeostasis through lipoprotein pathways can lead the depletion of sulfatides in myelin sheaths. ${ }^{56,57}$ On the other hand, the ceramide elevation in $A D$ was suggested to be a possible degradation product of sulfatides ${ }^{18}$, but other explanations include $A \beta$ induced membrane oxidative stress ${ }^{58}, A \beta$ mediated activation of sphingomyelinases (SMases) catalyzing the breakdown of SM to ceramide $^{59,60}$, and multiple gene expression abnormalities such as increased cerebral expression of genes involved in ceramide de novo synthesis along with the reduced expression of genes reguired for glycosphingolipid synthesis out of ceramide. ${ }^{61}$ Our data suggest both sulfatide depletion and ceramide elevation are amyloid plaque-associated phenomena in tgArcSwe AD mice pathology (Figure 4m,n,p). Indeed, an earlier study performed by quantitative fluorescent staining revealed amyloid plaque-associated demyelination and oligodendrocyte loss in both human and transgenic mice AD-brain. ${ }^{62}$ Therefore, substantial amyloid plaque-associated depletion of sulfatides could reinforce the the previous results 
indicating amyloid plaques could be associated with regression of myelination which is another pathological hallmark AD.

Along with sulfatide depletion and ceramide elevation, substantial accumulation of gangliosides (GM3 18:0, GM3 20:0, GM2 18:0) were revealed on the same amyloid plaques (Figure 4j-I, o). Most earlier studies indicated a general reduction of gangliosides ${ }^{63-65}$ along with the elevation of simpler gangliosides (GM2, GM3) $)^{65,66}$ in both transgenic mice and human AD brain extracts, which was hypothesized to be a result of accelerated lysosomal degradation and/or astrogliosis during neuronal cell death. ${ }^{66}$ This in line with the reported accumulations of GM2 and GM3 in lysosomal storage disorders. ${ }^{67,68}$

Phospholipids are essential constituents of neuronal membranes providing structural integrity and functional properties. ${ }^{69}$ Moreover, lysophospholipids, metabolic intermediates of phospholipid metabolism ${ }^{70}$ interact with the lipid and protein moieties of neural membranes and modulate the function of neural membrane proteins, such as enzymes and growth factors. ${ }^{71}$ Along with the sphingolipids, alterations in the levels of phospholipids, Iysophospholipids along with the phospholipid-metabolizing enzymes such as PLA, PLC, and PLD were reported in post-mortem human and trangenic AD mice brain tissues. ${ }^{72-75}$

Despite the high abundance and the efficient ionization of many of them, the structural diversity of phospholipids and lysophospholipids limit their comprehensive screening within a single MALDI-IMS analysis. Therefore, subsequent dual polarity lipid MALDI-IMS on the same tissue section increases the number of detected lipid species which can be correlated to spatial histopathological features. ${ }^{31,36,76}$ Here, high spatial resolution, dual polarity analysis on the hippocampus of tgArcSwe mice brain tissue allowed us to simultaneously correlate the alterations of phospholipid (Figure 5) and lysophospholipid (Figure 6) species to microscopic amyloid plaque structures. In detail, lysophosphatidic acids (LPA), phosphatidic acids (PA), lysophosphatidylethanolamine (LPE), phosphatidylethanolamines (PE), phosphatidylserines (PS), lysophosphatidylinositols (LPI) and phosphatidylinositols (PI) are observed in negative ion mode can be correlated lysophosphatidylcholines (LPC) and phosphatidylcholines (PC) which are observed in positive ion mode on the same pixel points. (Figure 5,6) Inspection of 
ion images in phsopholipid region revealed depletion of PE (40:6 m/z 790.6), PE-p (40:6 m/z 774.6), PS (40:6 m/z 834.6) species along with the elevation of PA (32:0, $\mathrm{m} / \mathrm{z} 647.5)$, PENMe2 (32:0 m/z 718.5), PC (32:0 m/z 734.6), PE (36:1 744.5), PI (36:4 m/z 857.6), PI (38:5 $\mathrm{m} / \mathrm{z}$ 883.6), $\mathrm{PI}$ (38:4 m/z 885.6) species on the amyloid plaque regions (Figure 5). Interestingly, PA (34:2 m/z 671.5) species revealed inconsistent distributions. Heteregoneous elevation and depletion of them were observed on the amyloid plaque regions (Figure 5c).

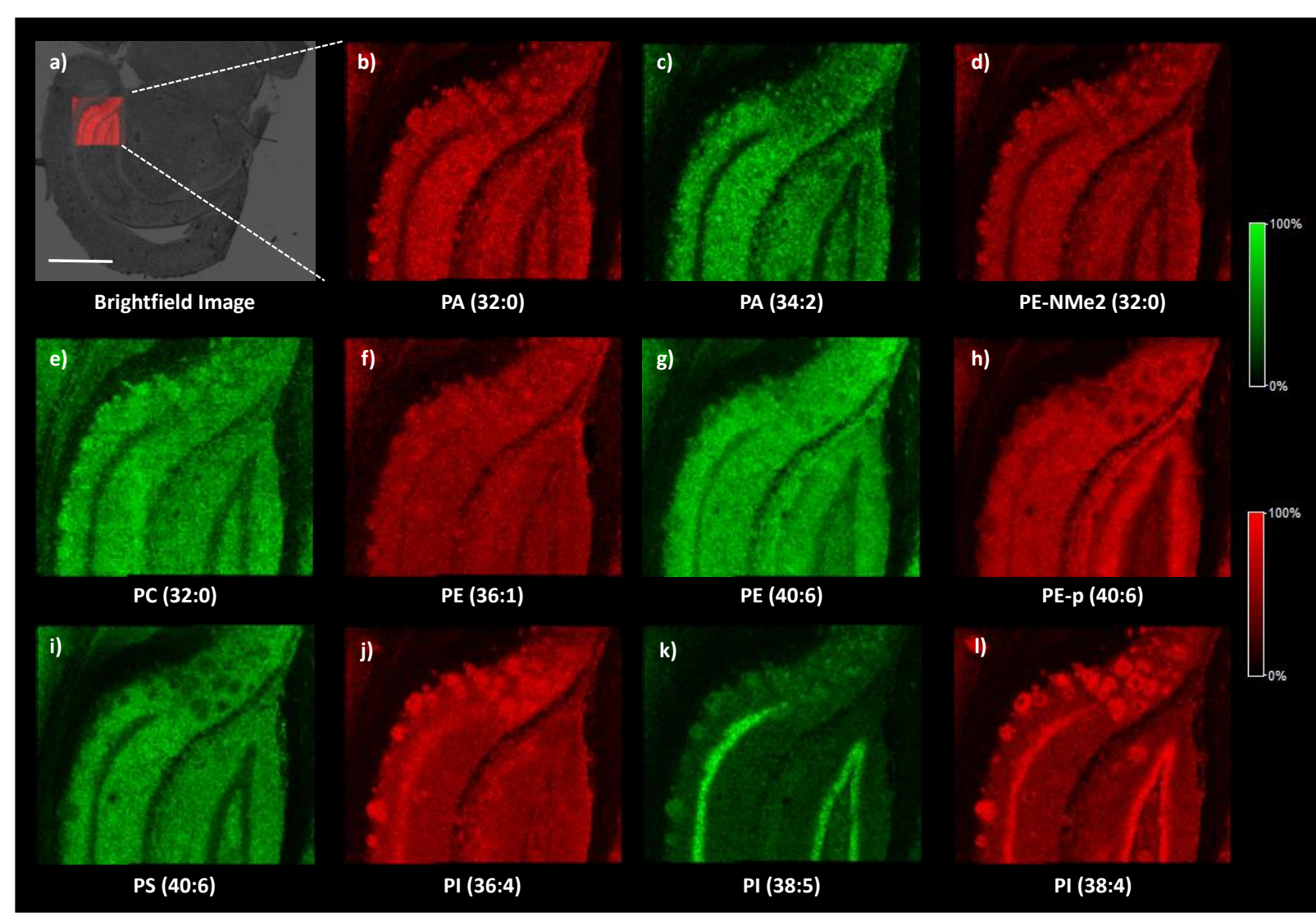

Figure 5. Amyloid plaque-associated alterations of phospholipids including phosphatidic acids (PA), phosphatidylethanolamines (PE), phosphatidylcholines (PC), phosphatidylserines (PS) and phosphatidylinositols $(\mathrm{PI})$ were revealed by dual polarity MALDI-IMS on the same pixel points. a) brightfield image of coronal brain tissue section of tgArcSwe, b) PA (32:0, m/z 647.5), c) PA (34:2 m/z 671.5), d) PE-NMe2 (32:0 m/z 718.5 ), e) PC (32:0 m/z 734.6), f) PE (36:1 744.5), g) PE (40:6 m/z 790.6), h) PE-p (40:6 m/z 774.6), i) PS (40:6 m/z 834.6), j) PI (36:4 m/z 857.6), k) PI (38:5 m/z 883.6) and I) PI (38:4 m/z 885.6). Scale bar: $2 \mathrm{~mm}$. 
Early reports generally indicated reductions of PE-PLs, PC-PLs and PI-PLs in human postmortem and transgenic $A D$-brains ${ }^{17,72,77,78}$ which was speculated to be due to the increased oxidative stress and stimulated activity of phospholipases in the presence of $A \beta$ peptides resulting in $\mathrm{PL}$ degradation. ${ }^{75,79,80}$ Our data indicates a clear depletion of phospholipids containing polyunsaturated fatty acids (PUFA) in their structure including PE (40:6 m/z 790.6), PE-p (40:6 m/z 774.6), PS (40:6 m/z 834.6) on the amyloid plaques which might be a result of lipid peroxidation by amyloid plaque-associated oxidative stress as previously demonstrated. ${ }^{80-}$ ${ }^{82}$ On the other hand, PA (32:0, m/z 647.5), PE-NMe2 (32:0 m/z 718.5), PC (32:0 m/z 734.6), PE (36:1 744.5) species containing no or few unsaturated fatty acids in their structure were elevated on amyloid plaques which can be explained by the possible binding of these lipids to amyloid peptides during amyloid oligomerization. ${ }^{83}$ In contrast to earlier reports on brain tissue extracts $^{72}$, phosphatidylinositol including PI (36:4 m/z 857.6), PI (38:5 m/z 883.6), PI (38:4 m/z 885.6) were elevated and localized to amyloid plaques, irrespective of their FA moeity (Figure $5 \mathrm{j}, \mathrm{k}, \mathrm{I})$. While this can be due to the pathogenesis difference between human and transgenic mice AD-brains, our results represents the alterations of this species in spatially confined amyloid plaques. AD-associated reduction of phosphatidylinositol kinase activity and depletion of phosphoinositide species in human AD-brain tissue were previously reported..$^{72,84}$ Furthermore, oligomeric $A \beta$ peptide was demonstrated to hinder phosphatidylinositol-4,5biphosphate levels in neurons. ${ }^{11}$ Therefore, elevation of phosphatidylinositols on amyloid plaques might be associated with disruption of phosphoinositide formation pathways.

Along with the amyloid plaque-associated alterations of phospholipids, inspection of ion images revealed accumulation of lysophospholipids including LPA (16:0, m/z 409.3), LPA (18:1, m/z 435.3), LPA (18:0, m/z 437.3), ) LPE (16:0, m/z 452.5), LPE (18:0, m/z 480.5), LPC (16:0, m/z 496.3), ) LPC (18:1, m/z 522.3), LPC $(18: 0,524.3)$ to amyloid plaques. (Figure 6) Elevation of LPC species were previously demonstrated in the brains of 9 month-old transgenic AD mice (5XFAD) using MALDI-IMS. ${ }^{75}$ Further, immunostaining of amyloid peptides and PLA 2 indicated an extensive accumulation of amyloid peoptides in the cortex and subiculum along with the partial accumulation of $\mathrm{PLA}_{2}$ around the $A \beta$ plaques. ${ }^{75}$ Therefore, this suggest an 
amyloid plaque-associated hyperactivity of $\mathrm{PLA}_{2}$ which can result in the accumulation of Iysophospholipids. ${ }^{85}$ Our high spatial resolution reveals that lysophospholipid accumulation is amyloid plaque-associated phenomenon in tgArcSwe $A D$ mice pathology which might be explained by the stimulated activity of phospholipases around amyloid plaques.

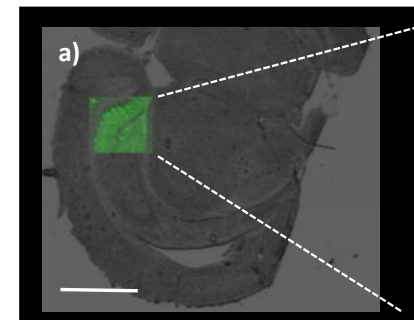

Brightfield Image

e)

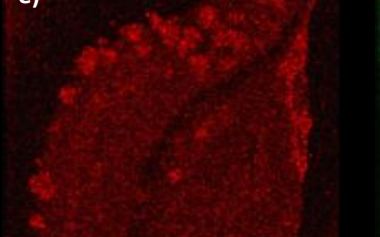

LPE (16:0)

i)

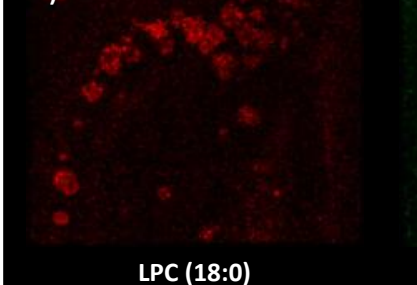

b)

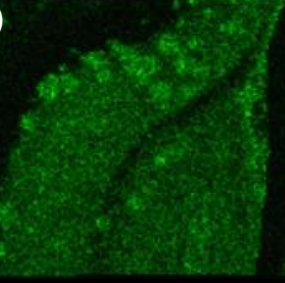

LPA (16:0)

f)

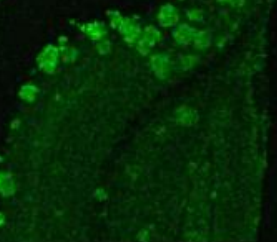

LPE (18:0) c)

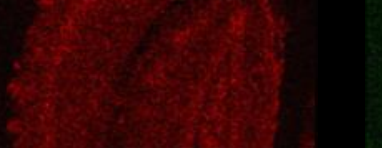

LPA (18:1)

g)

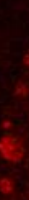

j) d)

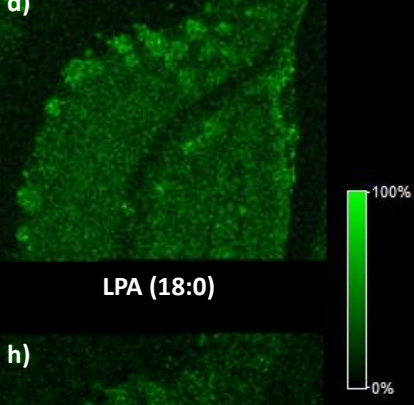

Figure 6. Amyloid plaque-associated alterations of lysophospholipids including lysophosphatidic acids (LPA), lysophoshatidylethanolamines (LPE), lysophosphatidylcholines (LPC) and lysophosphatidyinositols (LPI) were revealed by dual polarity MALDI-IMS on the same pixel points. a) brighfield image of the coronal brain tissue section of tgArcSwe. b) LPA (16:0, m/z 409.3), c) LPA( 18:1, m/z 435.3), d) LPA (18:0, m/z 437.3), e) LPE (16:0, m/z 452.5), f) LPE (18:0, m/z 480.5), g) LPC (16:0, $\mathrm{m} / \mathrm{z}$ 496.3), h) LPC (18:1 m/z 522.3), i) LPC (18:0 524.3), j) LPC (20:4, m/z 544.3), k) LPI (16:0, 571.3), I) LPI (18:0, m/z 599.3). Scale bar: $2 \mathrm{~mm}$

\section{CONCLUSIONS}


In summary, we have applied a multimodal MALDI-IMS method to reveal highthroughput molecular information on amyloid plaques at high spatial resolutions in 18 month-old transgenic (tgArcSwe) AD mouse brains. Inspection of ion images revealed plaque-associated accumulation of amyloid peptides isoforms and neuronal lipids at high spatial resolutions. Dual polarity MALDI-IMS analysis of lipids revealed high troughput lipid molecular information which can be correlated to individual amyloid plaques at 10 um spatial resolution. Here, we were, for the first time, able to correlate amyloid plaque-associate alterations of several sphingolipids, phospholipids and lysophospholipids to individual plaque features and certain amyloid peptide isoforms on the same tissue section. In conclusion, this methodology substantially increased the spatial amyloid plaque-associated molecular information. Insights into the possible pathways for the plaque-associated alterations of lipids were discussed.

AUTHOR CONTRBUTIONS. I.K. and J.H. conceived and designed the study. I.K. designed and performed MALDI-IMS experiments and wrote the paper. All authors revised the paper.

FUNDING The Swedish Research Council VR (\#2014-6447, J.H.; \#2012- 1593, S.S.; \#20132546, H.Z.; \#2013-14002, K.B.), the European Research Council (\#681712, H.Z.), Marie Sklodowska Curie Actions (Cofund, Project INCA 600398, J.H.), the Royal Society of Arts and Sciences in Gothenburg (KVVS, J.H.), Alzheimerfonden (J.H., K.B., S.S., D.S.), Demensfonden (J.H.), Hjärnfonden (K.B., S.S.), Jeanssons Stiftelsen (J.H.), Ahlen Stiftelsen (J.H., S.S., D.S.), Svenska Lundbeck-stiftelsen'(D.S.), Stiftelsen Gamla Tjänarinnor (J.H., D.S., K.B.), Stohnes Stiftelse (J.H., S.S., D.S.), and Stiftelsen Wilhelm och Martina Lundgrens Vetenskapsfond (J.H.) are acknowledged for financial support.

NOTES The authors declare no competing financial interest. 
ACKNOWLEDGMENTS MALDI-IMS experiments were performed the go:IMS imaging MS infrastructure at the University of Gothenburg (www.go-ims.gu.se) headed by Prof. Andrew Ewing.

LIST OF ABBREVIATIONS AD, Alzheimer's disease; $A \beta$, amyloid beta; Cer, ceramides; FA, formic acid; IHC, immunohistochemistry; IMS, imaging mass spectrometry; ITO, indium tinoxide; LPC, lysophosphatidylcholine; MALDI, matrix-assisted laser desorption/ionization; OCT, optimal cutting temperature; PC, phosphatidylcholine; PE, phosphatidylethanolamine; PI, phosphatidylinositols; PG, phosphatidylglycerol; PS, phosphatidylserine; RN, reflector negative mode; RMS, root-mean-square; ROI, region of interest; RP, reflector positive mode; SM, sphingomyelin; ST, sulfatides; TOF, time-of-flight.

\section{REFERENCES}

(1) Ballatore, C.; Lee, V. M.-Y.; Trojanowski, J. Q. Nature Reviews Neuroscience 2007, 8, 663-672.

(2) Hardy, J. A.; Higgins, G. A. Science 1992, 256, 184.

(3) Foley, P. Biochimica et Biophysica Acta (BBA)-Molecular and Cell Biology of Lipids 2010, 1801, 750753.

(4) Alzheimer, A. Allgemeine Zeitschrife Psychiatrie 1907, 64, 146-148.

(5) Hardy, J.; Selkoe, D. J. science 2002, 297, 353-356.

(6) Sakono, M.; Zako, T. The FEBS journal 2010, 277, 1348-1358.

(7) Haass, C.; Selkoe, D. J. Nature reviews Molecular cell biology 2007, 8, 101-112.

(8) Di Paolo, G.; Kim, T.-W. Nature Reviews Neuroscience 2011, 12, 284-296.

(9) Cheng, H.; Vetrivel, K. S.; Gong, P.; Meckler, X.; Parent, A.; Thinakaran, G. Nature Clinical Practice Neurology 2007, 3, 374-382.

(10) Sanchez-Mejia, R. O.; Newman, J. W.; Toh, S.; Yu, G.-Q.; Zhou, Y.; Halabisky, B.; Cissé, M.; Scearce-Levie, K.; Cheng, I. H.; Gan, L. Nature neuroscience 2008, 11, 1311-1318.

(11) Berman, D. E.; Dall'Armi, C.; Voronov, S. V.; McIntire, L. B. J.; Zhang, H.; Moore, A. Z.;

Staniszewski, A.; Arancio, O.; Kim, T.-W.; Di Paolo, G. Nature neuroscience 2008, 11, 547-554.

(12) Yanagisawa, K.; Odaka, A.; Suzuki, N.; Ihara, Y. Nature medicine 1995, 1, 1062-1066.

(13) Bu, G. Nature Reviews Neuroscience 2009, 10, 333-344.

(14) Selkoe, D. J. Neuron 1991, 6, 487-498.

(15) Grimm, M. O.; Mett, J.; Grimm, H. S.; Hartmann, T. Frontiers in Molecular Neuroscience 2017, 10.

(16) Panchal, M.; Loeper, J.; Cossec, J.-C.; Perruchini, C.; Lazar, A.; Pompon, D.; Duyckaerts, C. Journal of lipid research 2010, 51, 598-605.

(17) Han, X.; Holtzman, D. M.; McKeel, D. W. Journal of neurochemistry 2001, 77, 1168-1180.

(18) Han, X.; M Holtzman, D.; W McKeel, D.; Kelley, J.; Morris, J. C. Journal of neurochemistry 2002, 82, 809-818.

(19) Portelius, E.; Bogdanovic, N.; Gustavsson, M. K.; Volkmann, I.; Brinkmalm, G.; Zetterberg, H.; Winblad, B.; Blennow, K. Acta neuropathologica 2010, 120, 185-193. 
(20) Sullivan, P.; Han, B.; Liu, F.; Mace, B.; Ervin, J.; Wu, S.; Koger, D.; Paul, S.; Bales, K. Neurobiology of aging 2011, 32, 791-801.

(21) McDonnell, L. A.; Heeren, R. Mass spectrometry reviews 2007, 26, 606-643.

(22) Stoeckli, M.; Staab, D.; Staufenbiel, M.; Wiederhold, K.-H.; Signor, L. Analytical biochemistry 2002, 311, 33-39.

(23) Kelley, A. R.; Perry, G.; Castellani, R. J.; Bach, S. B. ACS chemical neuroscience 2016, 7, 261-268.

(24) Solé-Domènech, S.; Sjövall, P.; Vukojević, V.; Fernando, R.; Codita, A.; Salve, S.; Bogdanović, N.; Mohammed, A. H.; Hammarström, P.; Nilsson, K. P. R. Acta neuropathologica 2013, 125, 145-157.

(25) Carlred, L.; Gunnarsson, A.; Solé-Domènech, S.; Johansson, B. r.; Vukojević, V.; Terenius, L.; Codita, A.; Winblad, B.; Schalling, M.; Höök, F. Journal of the American Chemical Society 2014, 136, 9973-9981.

(26) Carlred, L.; Vukojević, V.; Johansson, B.; Schalling, M.; Höök, F.; Sjövall, P. Biointerphases 2016, $11,02 \mathrm{~A} 312$.

(27) Carlred, L.; Michno, W.; Kaya, I.; Sjövall, P.; Syvänen, S.; Hanrieder, J. Journal of neurochemistry 2016, 138, 469-478.

(28) Kakuda, N.; Miyasaka, T.; Iwasaki, N.; Nirasawa, T.; Wada-Kakuda, S.; Takahashi-Fujigasaki, J.; Murayama, S.; Ihara, Y.; Ikegawa, M. Acta neuropathologica communications 2017, 5, 73.

(29) Kaya, I.; Brinet, D.; Michno, W.; Syvänen, S.; Sehlin, D.; Zetterberg, H.; Blennow, K.; Hanrieder, J. r. ACS chemical neuroscience 2017, 8, 347-355.

(30) Kaya, I.; Michno, W.; Brinet, D.; lacone, Y.; Zanni, G.; Blennow, K.; Zetterberg, H.; Hanrieder, J. r. Analytical Chemistry 2017, 89, 4685-4694.

(31) Kaya, I.; Brinet, D.; Michno, W.; Başkurt, M.; Zetterberg, H.; Blenow, K.; Hanrieder, J. r. ACS chemical neuroscience 2017, 8, 2778-2790.

(32) Selkoe, D. J. Journal of Biological Chemistry 1996, 271, 18295-18298.

(33) Lord, A.; Kalimo, H.; Eckman, C.; Zhang, X.-Q.; Lannfelt, L.; Nilsson, L. N. Neurobiology of aging 2006, 27, 67-77.

(34) Yang, J.; Caprioli, R. M. Analytical chemistry 2011, 83, 5728-5734.

(35) Optimizing, U. Journal of Mass Spectrometry 2006, 41, 705-716.

(36) Thomas, A. I.; Charbonneau, J. L.; Fournaise, E.; Chaurand, P. Analytical chemistry 2012, 84, 2048-2054.

(37) Molin, L.; Seraglia, R.; Dani, F. R.; Moneti, G.; Traldi, P. Rapid Communications in Mass Spectrometry 2011, 25, 3091-3096.

(38) Carlred, L.; Michno, W.; Kaya, I.; Sjövall, P.; Syvänen, S.; Hanrieder, J. Journal of Neurochemistry 2016.

(39) Jackson, S. N.; Wang, H.-Y. J.; Woods, A. S.; Ugarov, M.; Egan, T.; Schultz, J. A. Journal of the American Society for Mass Spectrometry 2005, 16, 133-138.

(40) Kojro, E.; Gimpl, G.; Lammich, S.; März, W.; Fahrenholz, F. Proceedings of the National Academy of Sciences 2001, 98, 5815-5820.

(41) Grimm, M. O.; Mett, J.; Stahlmann, C. P.; Grösgen, S.; Haupenthal, V. J.; Blümel, T.; Hundsdörfer, B.; Zimmer, V. C.; Mylonas, N. T.; Tanila, H. Frontiers in aging neuroscience 2015, 7.

(42) Grimm, M. O.; Grösgen, S.; Rothhaar, T. L.; Burg, V. K.; Hundsdörfer, B.; Haupenthal, V. J.; Friess, P.; Müller, U.; Fassbender, K.; Riemenschneider, M. International Journal of Alzheimer's Disease 2011, 2011.

(43) Grimm, M. O.; Kuchenbecker, J.; Rothhaar, T. L.; Grösgen, S.; Hundsdörfer, B.; Burg, V. K.; Friess, P.; Müller, U.; Grimm, H. S.; Riemenschneider, M. Journal of neurochemistry 2011, 116, 916-925.

(44) Grimm, M. O.; Hundsdörfer, B.; Grösgen, S.; Mett, J.; Zimmer, V. C.; Stahlmann, C. P.; Haupenthal, V. J.; Rothhaar, T. L.; Lehmann, J.; Pätzold, A. Cellular Physiology and Biochemistry 2014, 34, 92-110.

(45) Grimm, M. O.; Grimm, H. S.; Pätzold, A. J.; Zinser, E. G.; Halonen, R.; Duering, M.; Tschäpe, J.-A.; De Strooper, B.; Müller, U.; Shen, J. Nature cell biology 2005, 7, 1118-1123.

(46) Tamboli, I. Y.; Prager, K.; Barth, E.; Heneka, M.; Sandhoff, K.; Walter, J. Journal of Biological Chemistry 2005, 280, 28110-28117. 
(47) Onodera, T.; Futai, E.; Kan, E.; Abe, N.; Uchida, T.; Kamio, Y.; Kaneko, J. The Journal of Biochemistry 2014, 157, 301-309.

(48) Sheikh, A. M.; Michikawa, M.; Kim, S.; Nagai, A. Neuroscience 2015, 292, 159-169.

(49) McLaurin, J.; Franklin, T.; Chakrabartty, A.; Fraser, P. Journal of molecular biology 1998, 278, 183194.

(50) Hannun, Y. A.; Obeid, L. M. Nature reviews Molecular cell biology 2008, 9, 139-150.

(51) He, X.; Huang, Y.; Li, B.; Gong, C.-X.; Schuchman, E. H. Neurobiology of aging 2010, 31, 398-408.

(52) Haughey, N. J.; Bandaru, V. V.; Bae, M.; Mattson, M. P. Biochimica et Biophysica Acta (BBA)-

Molecular and Cell Biology of Lipids 2010, 1801, 878-886.

(53) Hirahara, Y.; Wakabayashi, T.; Mori, T.; Koike, T.; Yao, I.; Tsuda, M.; Honke, K.; Gotoh, H.; Ono, K.; Yamada, H. Journal of neurochemistry 2017, 140, 435-450.

(54) Marcus, J.; Honigbaum, S.; Shroff, S.; Honke, K.; Rosenbluth, J.; Dupree, J. Glia 2006, 53, 372-381.

(55) Han, X.; Cheng, H.; Fryer, J. D.; Fagan, A. M.; Holtzman, D. M. Journal of Biological Chemistry

2003, 278, 8043-8051.

(56) Cheng, H.; Zhou, Y.; Holtzman, D. M.; Han, X. Neurobiology of aging 2010, 31, 1188-1196.

(57) Han, X. Journal of neurochemistry 2007, 103, 171-179.

(58) Cutler, R. G.; Kelly, J.; Storie, K.; Pedersen, W. A.; Tammara, A.; Hatanpaa, K.; Troncoso, J. C.;

Mattson, M. P. Proceedings of the National Academy of Sciences 2004, 101, 2070-2075.

(59) Jana, A.; Pahan, K. Journal of Biological Chemistry 2004, 279, 51451-51459.

(60) Lee, J.-T.; Xu, J.; Lee, J.-M.; Ku, G.; Han, X.; Yang, D.-I.; Chen, S.; Hsu, C. Y. The Journal of cell biology 2004, 164, 123-131.

(61) Katsel, P.; Li, C.; Haroutunian, V. Neurochemical research 2007, 32, 845-856.

(62) Mitew, S.; Kirkcaldie, M. T.; Halliday, G. M.; Shepherd, C. E.; Vickers, J. C.; Dickson, T. C. Acta neuropathologica 2010, 119, 567-577.

(63) Svennerholm, L.; Gottfries, C. G. Journal of neurochemistry 1994, 62, 1039-1047.

(64) Crino, P. B.; Ullman, M. D.; Vogt, B. A.; Bird, E. D.; Volicer, L. Archives of neurology 1989, 46, 398401.

(65) Barrier, L.; Ingrand, S.; Damjanac, M.; Bilan, A. R.; Hugon, J.; Page, G. Neurobiology of aging 2007, 28, 1863-1872.

(66) Kracun, I.; Kalanj, S.; Talan-Hranilovic, J.; Cosovic, C. Neurochemistry international 1992, 20, 433438.

(67) Kreutz, F.; dos Santos Petry, F.; Camassola, M.; Schein, V.; Guma, F. C.; Nardi, N. B.; Trindade, V. M. T. Gene 2013, 527, 109-114.

(68) Dufresne, M.; Guneysu, D.; Patterson, N. H.; Marcinkiewicz, M. M.; Regina, A.; Demeule, M.; Chaurand, P. Analytical and Bioanalytical Chemistry 2016, 1-9.

(69) Farooqui, A. A.; Horrocks, L. A.; Farooqui, T. Chemistry and physics of lipids 2000, 106, 1-29.

(70) Farooqui, A. A.; Horrocks, L. A.; Farooqui, T. Journal of Molecular Neuroscience 2000, 14, 123 135.

(71) Kern, R.; Joseleau-Petit, D.; Chattopadhyay, M. K.; Richarme, G. Biochemical and biophysical research communications 2001, 289, 1268-1274.

(72) Stokes, C. E.; Hawthorne, J. N. Journal of neurochemistry 1987, 48, 1018-1021.

(73) Oliveira, T. G.; Di Paolo, G. Biochimica et Biophysica Acta (BBA)-Molecular and Cell Biology of Lipids 2010, 1801, 799-805.

(74) Ross, B. M.; Moszczynska, A.; Erlich, J.; Kish, S. J. Journal of neurochemistry 1998, 70, 786-793.

(75) Hong, J. H.; Kang, J. W.; Kim, D. K.; Baik, S. H.; Kim, K. H.; Shanta, S. R.; Jung, J. H.; Mook-Jung, I.; Kim, K. P. Journal of lipid research 2016, 57, 36-45.

(76) Ellis, S. R.; Cappell, J.; Potočnik, N. O.; Balluff, B.; Hamaide, J.; Van der Linden, A.; Heeren, R. M. Analyst 2016.

(77) Ginsberg, L.; Rafique, S.; Xuereb, J. H.; Rapoport, S. I.; Gershfeld, N. L. Brain research 1995, 698, 223-226.

(78) Igarashi, M.; Ma, K.; Gao, F.; Kim, H.-W.; Rapoport, S. I.; Rao, J. S. Journal of Alzheimer's Disease 2011, 24, 507-517. 
(79) Sanchez-Mejia, R. O.; Newman, J. W.; Toh, S.; Yu, G.-Q.; Zhou, Y.; Halabisky, B.; Cissé, M.; Scearce-Levie, K.; Cheng, I. H.; Gan, L. Nature neuroscience 2008, 11, 1311.

(80) Butterfield, D. A.; Drake, J.; Pocernich, C.; Castegna, A. Trends in molecular medicine 2001, 7, 548-554.

(81) Butterfield, D. A.; Lauderback, C. M. Free Radical Biology and Medicine 2002, 32, 1050-1060.

(82) Furman, R.; Murray, I. V.; Schall, H. E.; Liu, Q.; Ghiwot, Y.; Axelsen, P. H. ACS chemical neuroscience 2016, 7, 367-377.

(83) Avdulov, N. A.; Chochina, S. V.; Igbavboa, U.; Warden, C. S.; Vassiliev, A. V.; Wood, W. G. Journal of neurochemistry 1997, 69, 1746-1752.

(84) Jolles, J.; Bothmer, J.; Markerink, M.; Ravid, R. Journal of neurochemistry 1992, 58, 2326-2329.

(85) Kudo, I.; Murakami, M. Prostaglandins \& other lipid mediators 2002, 68, 3-58. 